\title{
The Appropriateness of Testing Platelet Factor 4/Heparin Antibody in Patients Suspected of Heparin-induced Thrombocytopenia
}

\author{
Vishal Jindal $^{1}$, Aditi Singh ${ }^{1}$, Ahmad D. Siddiqui ${ }^{2}$, Laszlo Leb ${ }^{2}$ \\ 1. Internal Medicine, St. Vincent Hospital, Worcester, USA 2. Hematology and Oncology, St. Vincent Hospital, \\ Worcester, USA
}

Corresponding author: Vishal Jindal, vishaljindal87@gmail.com

\begin{abstract}
Heparin-induced thrombocytopenia (HIT) is an adverse reaction to the administration of heparin due to the activation of the platelets by the immunoglobulin G (IgG) antibody-platelet factor 4 (PF4)/heparin immune complex. Since the clinical outcome is uncertain (as it could be associated with significant morbidity and sometimes death), an early diagnosis and appropriate treatment are necessary. The 4Ts pretest clinical scoring system and testing for all anti-PF4/heparin antibodies can markedly improve the diagnosis and prompt adequate treatment. Our study was undertaken to retrospectively evaluate the appropriateness of ordering the PF4 enzyme-linked immunosorbent assay (ELISA) test by using the 4Ts scoring system in a tertiary institution. We examined a database of 118 patients who had the PF4 ELISA test and calculated their 4Ts scores retrospectively. A total of 107 patients were evaluated; 95 patients $(88.79 \%)$ had a negative PF4 ELISA assay and 12 patients tested positive (11.21\%). Only one patient tested weakly positive in the low probability group (negative predictive value $98 \%$ ). In the intermediate group, six patients were strongly positive (optical density $(\mathrm{OD})>1.0$ ). In this latter group, further confirmatory testing using serotonin release assays (SRAs) could have been done. We also evaluated the setting where the tests were performed and found that the majority of patients (63.55\%) were tested in the intensive care unit (ICU) where thrombocytopenia is multifactorial. We concluded that the large majority of patients were not appropriately evaluated prior to testing, which incurred unnecessary expense and patient distress. For the proper identification of patients suspected of HIT who should undergo PF4/heparin antibody testing, further education of the ordering physicians is recommended.
\end{abstract}

Categories: Internal Medicine, Other

Keywords: 4ts scoring, platelet factor 4 (pf4), pf4 elisa test, heparin-induced thrombocytopenia (hit)

\section{Introduction}

Heparin-induced thrombocytopenia (HIT) is an important and serious complication of heparin treatment. The first case of arterial thrombosis associated with heparin treatment was described in the 1950's [1];

Received 10/16/2018

Review began 10/21/2018 Review ended 10/29/2018 Published 10/31/2018

\section{() Copyright 2018}

Jindal et al. This is an open access article distributed under the terms of the Creative Commons Attribution License CC-BY 3.0., which permits unrestricted use, distribution, and reproduction in any medium, provided the original author and source are credited. however, thrombocytopenia as a common occurrence in patients treated with heparin was recognized only in 1970 [2]. In the late 1970's, the clinical features of HIT were defined [3]. The demonstration that HIT is caused by platelet factor 4 (PF4)/heparin antigen-antibody complexes [4-5] led to the development of a PF4 enzyme-linked immunosorbent assay (PF4-ELISA) and to the more specific serotonin release functional assay. The evaluation of the clinical probability of HIT was significantly improved by introducing in practice the 4 Ts scoring system, which has a high negative predictive value [6].

The easy availability of an automated platelet count prompted physicians to suspect excessive HIT in heparinized patients who developed thrombocytopenia. Furthermore, the PF4-ELISA assay, being relatively easily obtainable, was used to confirm the diagnosis. In the last decade, the almost universally applied 4Ts scoring system and the demonstration of the low specificity of PF4-ELISA assay were expected to further improve the diagnosis of clinically suspected HIT. The present study was undertaken to retrospectively evaluate the appropriateness of ordering the PF4 ELISA test in clinically suspected HIT by using the 4Ts scoring system as a pretest tool in a tertiary hospital. We hope that this study will further contribute to a more judicious diagnosis and management of patients suspected of HIT.

\section{Materials And Methods}

In this study, we did a retrospective chart review of patients at Saint Vincent Hospital, Worcester who had a PF4 ELISA assay done for HIT during the in-hospital stay. After the Metrowest Medical Center Institutional Review Board approval, a total of 118 hospitalized patients from January 1, 2015 to June 30, 2017 were enrolled in this study. Out of that, 11 patients were removed - eight due to the lack of clinical data and three due to doubling, as they had a repeat test within three months. A chart review, including laboratory data and radiological data, was used to calculate the fall in the platelet count, exposure to heparin, any thrombotic event, and the presence of other causes of thrombocytopenia. From this data, we calculated the 4Ts score and divided patients into three groups: low probability - 4Ts score 0 to 3, intermediate probability - 4Ts 


\section{Cureus}

score 4 - 5, and high probability - 4Ts score $\geqslant 6$. The PF4 ELISA antibody results with optical density (OD) values were divided into three groups, 0 to 0.4 (negative), 0.4 to 1 (weakly positive), and $\geqslant 1$ (strongly positive). Along with that, additional data was integrated regarding the demographics, diagnosis, and site of admission (Table 1). The PF4 ELISA assay for heparin antibodies was performed according to the manufacturer's specifications.

\begin{tabular}{|c|c|}
\hline & Mean \\
\hline Age & $71.25 \pm 11.81$ \\
\hline Gender & Frequency (percentage) \\
\hline Male & $52(48.60 \%)$ \\
\hline Female & $55(51.40 \%)$ \\
\hline \multicolumn{2}{|l|}{ Major Diagnosis } \\
\hline Sepsis & $40(37.38 \%)$ \\
\hline Malignancy & $14(13.08 \%)$ \\
\hline MI/CABG/cardiogenic shock & $15(14.02 \%)$ \\
\hline Intestinal perforation & $10(9.35 \%)$ \\
\hline Renal failure & $21(19.63 \%)$ \\
\hline Pneumonia and other respiratory issues & $26(24.30 \%)$ \\
\hline Others & $10(9.35 \%)$ \\
\hline \multicolumn{2}{|l|}{ Settings } \\
\hline ICU & $68(63.55 \%)$ \\
\hline Non-ICU & 39 (36.45\%) \\
\hline
\end{tabular}

TABLE 1: Demographics, Diagnosis, and Setting

CABG: coronary artery bypass grafting; ICU: intensive care unit; MI: myocardial infarction

\section{Results}

\section{Demographic data}

A total of 118 patient's profiles were analyzed retrospectively, and out of that number, 11 were excluded from the study. Eight patients were excluded due to the lack of data and three due to doubling as they had a repeat test within three months. Thus, our total sample size was 107. In the study population, the mean age was $71.25 \pm 11.81$, with a minimum age of 43 and maximum age of 96 (95\% confidence interval (CI) 68.99 to 73.52) (Table 1). The total of male participants was 52 (48.60\%) and the remaining 55 (73.47\%) were female participants. Among the study population, 51 (47.66\%) were low probability, 51 (47.66\%) were intermediate probability, and five (4.67\%) were high probability.

\section{Intensive care unit (ICU) admission and diagnosis}

Sixty-eight out of 107 patients were in the ICU, and in further subgroup division, 30 (44\%) belonged to the low probability group, 36 (53\%) belonged to the intermediate probability group, and two (3\%) belonged to high probability group. The difference in the proportion of total 4Ts score group between the three subgroups in ICU was not statistically significant (P value - 0.249). Among the study population, 40 (37.38\%) had sepsis. The number of people with malignancy, MI/CABG/cardiogenic shock, intestinal perforation, renal failure, pneumonia and other respiratory issues, and other diagnosis was 14 (13.08\%), 15 (14.02\%), 10 (9.35\%), 21 (19.63\%), 26 (24.30\%), and 10 (9.35\%), respectively.

\section{PF4 ELISA HIT antibody and 4Ts scoring}

Out of the total sample size, 95 patients (88.79\%) were negative for the HIT antibody and only 12 patients $(11.21 \%)$ were positive $(\mathrm{OD}>0.40)$. The patients with the positive PF4 HIT ELISA antibody were further classified into two groups on the basis of the OD value: weakly positive (OD value: $0.40-0.99$ ) and strongly positive (OD: > 1.00). There were six patients (5.61\%) in each group, as shown in Table 2 . On comparing the 


\section{Cureus}

4Ts score with OD values, we found only one patient tested weakly positive in low probability in the 4Ts score group and the rest were negative, which gives a negative predictive value of the 4Ts scoring system in the low probability group of $98 \%$ (Figure 1 ). The other 11 patients were in the intermediate probability group, and there was no patient with a PF4-HIT antibody in the high probability group. There were only six patients who had an OD value of more than 1 . Therefore, the positive predictive value of this test was $19.6 \%$.

\begin{tabular}{|l|c|}
\hline Total 4Ts score group & $51(47.66 \%)$ \\
\hline Low probability $(0-3)$ & $51(47.66 \%)$ \\
\hline Intermediate probability $(4-5)$ & 5 \\
High probability $(\geq 6)$ & $(4.67 \%)$ \\
PF4 ELISA OD results & $95(88.79 \%)$ \\
$0-0.40$ (Negative) & $6(5.61 \%)$ \\
$0.41-0.99$ (weakly positive) & $6(5.61 \%)$ \\
$>1.0$ (Strongly positive)
\end{tabular}

TABLE 2: 4Ts Scoring and PF4 ELISA Results

OD: optical density; PF4-ELISA: platelet factor 4 enzyme-linked immunosorbent assay

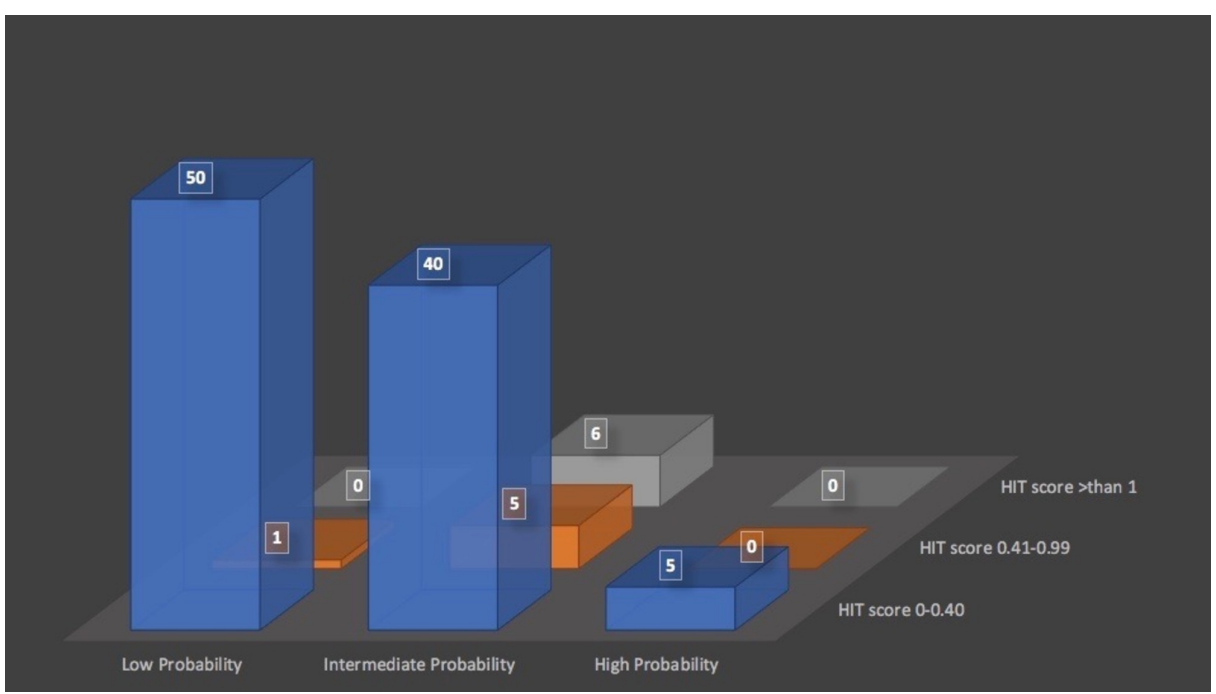

FIGURE 1: Comparison of 4Ts Score and PF4 ELISA Results

HIT: heparin-induced thrombocytopenia; PF4 ELISA: platelet factor 4 enzyme-linked immunosorbent assay

\section{Discussion}

The diagnosis of HIT is achieved by taking into account both clinical and laboratory data. The clinical pretest evaluation was improved by the use of the 4Ts clinical scoring system which divides patients into the low probability 4 Ts score $(0-3)$, intermediate probability $(4-5)$, and high probability $(\geqslant 6)$. Low 4 Ts scores were associated with a high negative predictive value (0.998) [7]. The intermediate and high scores had a poor positive predictive value for HIT ( 0.14 and 0.64, respectively) [7]. Based on these findings, the American Society of Haematology recommends against testing for HIT in patients with low pre-test probability but testing when the pre-test probability is $>8 \%$. This will include patients with intermediate or high 4 Ts score. The laboratory testing for HIT is performed in most hospitals using the polyspecific PF4 ELISA assay, which is widely available, offers a rapid turnaround time, and is highly sensitive (>99\%) but has a low specificity (30\% to $70 \%$ ) [8]. Using the quantification of the test with the OD value, it has been reported that the specificity improved significantly at the value of OD > 1.0 [9]. This strongly positive higher value correlates significantly with the "gold standard" serotonin release assays (SRAs). 
In this retrospective study, only 12 patients (11.21\%) out of 107 tested positive in the polyspecific PF4 ELISA assay having an OD value of $>0.40$, which is in the range of previously published studies at $3 \%$ and $17 \%[10$ 11]. However, only six patients (5.6\%) had a PF4 ELISA OD value > 1.0 which could correlate well the SRA and thus could be considered true positive. This value is still in the range of previous studies. In the low probability category of clinical suspicion, the negative predictive value was $98 \%$, which is similar to data published in the literature [11], and this is the group in which no testing should have been performed. Only six patients in the intermediate probability category of clinical suspicion for HIT tested positive with an OD value > 1.0, which shows the positive predictive value of the 4 Ts scoring system is low in patients of the intermediate probability group. Since the positive test in this group increases the post-test probability of HIT to $40 \%$ to 64\% [7], an SRA could have been indicated to confirm HIT or if the clinical condition necessitated administering direct thrombin inhibitors (DTI). Regarding the five patients in the high probability category of clinical suspicion in the 4Ts score, the positive predictive value is 0.64 and the negative PF4 ELISA assay was most likely due to the low number of tested patients. In case the suspicion for HIT remains high in such patients, they should probably be placed on DTI.

Regarding the setting of evaluated patients, the large majority of patients were from the ICU (63.45\%). This is of no surprise as in this setting many patients receive heparin treatment and they also often had thrombocytopenia. However, identification of the cause of thrombocytopenia in ICU is difficult as patients can develop thrombocytopenia as a result of multiple aetiologies, including liver disease, drugs, sepsis, or disseminated intravascular coagulation, making it difficult to attribute the low platelet count to heparin administration only. The fact that the number of beds in the ICU is relatively few and the patient turnover is low compared to the medical and surgical settings further emphasizes the disproportionate number of PF4 ELISA tests ordered in the ICU. The majority of patients were diagnosed with sepsis (38\%) and respiratory illnesses (24.30\%). All patients were recruited from the ICU, which further supports the difficulty to attribute the cause of thrombocytopenia to heparin treatment only.

\section{Conclusions}

Thus, this study confirms previous ones about the importance of implementing the 4Ts scoring system in clinical practice and limiting testing for PF4/heparin antibody assays only to patients with intermediate and high 4Ts values. By implementing such clinical guidelines, the number of ordered tests can be cut almost in half (which is also of economic value). It is of interest that none of the tested patients, particularly those with intermediate and high 4Ts, had their heparin therapy discontinued or changed to DTI. This was probably due to either non-reliance on the clinical evaluation or disregarding the test results. Our study demonstrates an insufficient education of the medical staff regarding the importance of pre-test clinical evaluation and the correct ordering of tests for PF4-heparin antibodies testing. Education of physicians regarding diagnosing and treating HIT is badly needed, particularly in the settings of ICU where the majority of inappropriate tests were ordered.

\section{Additional Information \\ Disclosures}

Human subjects: Consent was obtained by all participants in this study. Metrowest Medical Center Institutional Review Board, Framingham MA issued approval 2015-131. Animal subjects: All authors have confirmed that this study did not involve animal subjects or tissue. Conflicts of interest: In compliance with the ICMJE uniform disclosure form, all authors declare the following: Payment/services info: All authors have declared that no financial support was received from any organization for the submitted work. Financial relationships: All authors have declared that they have no financial relationships at present or within the previous three years with any organizations that might have an interest in the submitted work. Other relationships: All authors have declared that there are no other relationships or activities that could appear to have influenced the submitted work.

\section{References}

1. Weismann RE, Tobin RW: Arterial embolism occurring during systemic heparin therapy . AMA Arch Surg. 1958, 76:219-27. 10.1001/archsurg.1958.01280200041005

2. Rhodes GR, Dixon RH, Silver D: Heparin induced thrombocytopenia with thrombotic and hemorrhagic manifestations. Surg Gynecol Obstet. 1973, 136:409-16.

3. Babcock RB, Dumper CW, Scharfman WB: Heparin-induced immune thrombocytopenia. N Engl J Med. 1976, 295:237-41. 10.1056/NEJM197607292950501

4. Brandt S, Krauel K, Gottschalk KE, et al.: Characterisation of the conformational changes in platelet factor 4 induced by polyanions: towards in vitro prediction of antigenicity. Thromb Haemost. 2014, 112:53-64. 10.1160/TH13-08-0634

5. Ziporen L, Li ZQ, Park KS, et al.: Defining an antigenic epitope on platelet factor 4 associated with heparininduced thrombocytopenia. Blood. 1998, 92:3250-59.

6. Lo GK, Juhl D, Warkentin TE, et al.: Evaluation of pretest clinical score (4 T's) for the diagnosis of heparininduced thrombocytopenia in two clinical settings. J Thromb Haemost. 2006, 4:759-65. 10.1111/j.15387836.2006.01787.x

7. Cuker A, Gimotty PA, Crowther MA, Warkentin TE: Predictive value of the 4Ts scoring system for heparininduced thrombocytopenia: a systematic review and meta-analysis. Blood. 2012, 120:4160-67. 


\section{Cureus}

10.1182/blood-2012-07-44305

8. Jang IK, Hursting MJ: When heparins promote thrombosis: review of heparin-induced thrombocytopenia . Circulation. 2005, 111:2671-83. 10.1161/CIRCULATIONAHA.104.518563

9. Warkentin TE, Sheppard JI, Moore JC, et al.: Quantitative interpretation of optical density measurements using PF4-dependent enzyme-immunoassays. J Thromb Haemost. 2008, 6:1304-12. 10.1111/j.15387836.2008.03025.x

10. Warkentin TE: How I diagnose and manage HIT . Hematology Am Soc Hematol Educ Program. 2011, 2011:143-49. 10.1182/asheducation-2011.1.143

11. Bryant A, Low J, Austin S, Joseph JE: Timely diagnosis and management of heparin-induced thrombocytopenia in a frequent request, low incidence single centre using clinical 4T's score and particle gel immunoassay. Br J Haematol. 2008, 143:721-26. 10.1111/j.1365-2141.2008.07401.x 